\title{
Investigation of Pharmacological Mechanisms of Schisandrin a for the Treatment of Asthma Determined by Network Pharmacology and Experimental Validation
}

\section{Qing Qiu}

Guangdong Medical College Zhanjiang Campus: Guangdong Medical University

Fangfang Huang

Guangdong Medical College Zhanjiang Campus: Guangdong Medical University

Jiating Su

Guangdong Medical College Zhanjiang Campus: Guangdong Medical University

Qianwen Lin

Guangdong Medical College Zhanjiang Campus: Guangdong Medical University

\section{Yuge Huang}

Guangdong Medical College Zhanjiang Campus: Guangdong Medical University

lianxiang luo ( $\sim$ luolianxiang321@gdmu.edu.cn )

Guangdong Medical College Zhanjiang Campus: Guangdong Medical University https://orcid.org/0000-0002-3391-9713

\section{Research}

Keywords: Schisandrin A, Asthma, NF-kB, COX-2, Th2, Network pharmacology

Posted Date: August 12th, 2021

DOI: https://doi.org/10.21203/rs.3.rs-779304/v1

License: (c) (i) This work is licensed under a Creative Commons Attribution 4.0 International License. Read Full License 


\section{Abstract}

Traditional Chinese medicines (TCM) are increasingly applied and accepted in asthma prevention and treatment. In the present investigation, we aimed to evaluate the effects of schisandrin A against asthma and examine its underlying mechanism. Here, 68 intersection targets between schisandrin $A$ and asthma were identified by network pharmacology. Further enrichment analysis demonstrated that the nuclear factor-kappaB (NF-kB) signaling pathway may be a major signaling pathway and cyclooxygenase 2 (COX-2/PTGS2) may be a key target in the anti-asthmatic mechanism of schisandrin A. Then, the relevant mechanisms were verified. In vitro, we found that schisandrin A knock down the expression of COX-2 and iNOS (inducible nitric oxide synthase) in $16 \mathrm{HBE}$ cells and RAW264.7 cells in a dose-dependent manner. While, it ameliorated the epithelial barrier function injury, and reduced the activation of NF-kB signaling pathway effectively. Additionally, OVA-induced asthma mice model showed that inflammatory cell infiltration, mucus secretion as well as airway remodeling could be availably suppressed by schisandrin $A$ treatment. In conclusion, our data suggested that schisandrin A can reduce asthma symptoms by inhibiting inflammation production, including lowering the Th2 cell ratio, which provides a basis for further understanding of the treatment of asthma with schisandrin $A$.

\section{Introduction}

Asthma is a common chronic inflammatory disease characterized by airway hyperreactivity, airway inflammation, and airway remodeling [1]. The airways of asthmatic patients often show inflammatory cell infiltration, goblet cell hyperplasia, increased mucus secretion and epithelial cell tight junction changes [2]. T helper type 2 (Th2) lymphocytes activate eosinophils and mast cells in response to allergens by inducing the release of cytokines, which play an essential role in airway inflammation and excessive mucus secretion [3]. It is well-known that nuclear factor-kappaB (NF-kB) signaling pathway is an important pathway to regulate innate and adaptive immune responses $[4,5]$. Simultaneously, it is associated with asthma [6]. Cyclooxygenase 2 (COX-2), also known as prostaglandin-endoperoxide synthase 2 (PTGS2) and inducible nitric oxide synthase (iNOS) are involved in the activation of NF-KB [7]. On the other hand, the phosphorylation of NF-KB can increase the expression of iNOS and COX-2 $[8,9]$. Most of the current clinical treatments for asthma use western medicine treatments, such as corticosteroids and $\beta 2$ receptor agonists, but not all patients respond and larger doses of corticosteroids cause many side effects $[10,11]$. In recent years, traditional Chinese medicine (TCM) has been increasingly selected to treat asthma.

Schisandrin $A$ is a bioactive lignan isolated from the fruit of Schisandra chinensis, which has the effects of anti-inflammatory and anti-oxidation, that appears to be associated with the NF-KB signaling pathway $[12,13]$. Schisandrin $A$ has potential effects on osteoarthritis by inhibiting IL-1 $\beta$-induced inflammation and cartilage degradation through inhibition of MAPK and NF-KB signaling pathways [14]. In addition, Schisandrin A inhibits LPS-induced inflammatory and oxidative responses in RAW264.7 cells by inhibiting NF-KB, MAPK and PI3K/Akt pathways, indicating its potential role in over-activation of macrophages [15]. Studies have indicated that that schizandrin may be an effective novel therapeutic agent for the 
treatment of asthma, that appears to be mediated in part by a reduction in oxidative stress and airway inflammation [16-18]. Therefore, the purpose of our current study was to explore the protective effect of schisandrin A on asthma by in vitro and in vivo models.

\section{Materials And Methods}

\section{Construction of a Visual Network Map on the Target Collection of Chemical Components of schizandrin A}

The chemical composition structure, canonical SMILES and 2D structure (stored in SDF file format) of schizandrin A were obtained from the Pubchem database (https://pubchem.ncbi.nlm.nih.gov/). Then, the canonical SMILES was sent to the Swiss Target Prediction Database

(http://www.swisstargetprediction.ch/) to obtain the predicted targets of schisandrin A. As the same time, to ensure the completeness of targets collection, we used TTD database (Therapeutic Target Database, https://en.wikipedia.org/wiki/therapeutic-targets-database), CTD database (Comparative Toxicogenomics Database, http://ctdbase.org/), SEA database, BATMAN-TCM database and PharmMapper database (http://59.78.96.61/pharmmapper/) to collect the targets, and obtained more target genes of schisandrin A by means of literature search.

The target gene information were normalized using the Uniprot database (https://www.uniprot.org/) after removing duplicated genes.

\subsection{Target collection and screening of bronchial asthma}

Using "asthma" as the keyword in the GeneCards database (http://www.genecards.org/) and the OMIM database (Online Mendelian Inheritance in Man, http://www.omim.org/), asthma related target genes were acquired. The resulting target genes were combined, de-duplicated, and then normalized for target gene information using the Uniport database.

1. Construction of protein-protein interaction (PPI) network and acquisition of core targets of schizandrin $A$ in the treatment of bronchial asthma

The intersection target genes between schisandrin A and asthma were obtained by using Venny plot analysis (v2.1, http://bioinfogp.cnb.csic.es/tools/venny/index.html). The obtained intersection target genes were uploaded to the STRING database (https://string-db.org/) to obtain the protein-protein interaction (PPI) network, the species was defined as "Homo sapiens", and TSV file was saved. The PPI network was visualized using the Cytoscape (version 3.6.2) software, and the topological analysis of the PPI network was carried out by using the NetworkAnalyzer plugin for Cytoscape, thereby obtaining the topology indices of the network such as degree, betweeness centrality, closeness centrality, and average path length. Then, the hub genes from the PPI network were selected using the MCC algorithm of the cytoHubba plugin.

\subsection{GO functional enrichment and KEGG of core targets}


For functional enrichment analysis of the intersection target genes, we carried out Gene Ontology (GO) functional enrichment and Kyoto Encyclopedia of Genes and Genomes (KEGG) pathway analysis using the DAVID database (the Database for Annotation, Visualization and Integrated Discovery, http://david.abcc.ncifcrf.gov/). Furthermore, the results were screened using $P$ value $<0.05$, and the top ranked results were visualized.

\subsection{Cell cultures}

Human bronchial epithelial cell line (16 HBE) and murine macrophage cell line (RAW264.7) were purchased from Modification Type Culture Collection (ATCC, USA) and cultured in high glucose Dulbecco's Eagle's Medium (H-DMEM) medium supplemented with 10\% fetal bovine serum (FBS) and 1\% double antibody (penicillin and streptomycin) at $37^{\circ} \mathrm{C}$ in a humidified $5 \% \mathrm{CO}^{2}$ incubator.

\section{6 $16 \mathrm{HBE}$ and RAW264.7 cells grouping and treatment}

Plating was performed when growth was good and cells were confluent to approximately $80 \%-90 \% .16$ HBE cells were seeded in 12-well plates at $1.5 \times 105 \mathrm{cells} / \mathrm{ml}$, and divided into five groups: control, $\mathrm{D}$. pteronyssinus (HDM), HDM + schisandrin A $(1 \mu \mathrm{M}), \mathrm{HDM}+$ schisandrin $\mathrm{A}(5 \mu \mathrm{M})$ and $\mathrm{HDM}+$ schisandrin $\mathrm{A}$ $(10 \mu \mathrm{M})$. When the cells were $70-80 \%$ confluent, the medium was changed with serum-free medium for $12 \mathrm{~h}$. After 12h, different concentrations of schisandrin A (1, 5 and $10 \mu \mathrm{M}$, Baoji Herbest Bio-tech, China) were added for $0.5 \mathrm{~h}$. Then, HDM $(50 \mu \mathrm{g} / \mathrm{ml}$, Greer Laboratories, NC) was added for 24 hours, and then harvested for protein extraction. Meanwhile, RAW264.7 cells were seeded in 12-well plates at $2.0 \times 105$ cells/ml, and divided into five groups: control, lipopolysaccharide (LPS), LPS + schisandrin A $(1 \mu \mathrm{M})$, LPS + schisandrin $\mathrm{A}(5 \mu \mathrm{M})$ and LPS + schisandrin $\mathrm{A}(10 \mu \mathrm{M})$. After culturing overnight, different concentrations of schisandrin $A(1,5$ and $10 \mu \mathrm{M})$ were added for $0.5 \mathrm{~h}$, followed by LPS $(250 \mathrm{ng} / \mathrm{mL}$, Sigma-AldrichChina, China) for $24 \mathrm{~h}$, and then harvested for protein extraction. For immunofluorescence, 16 HBE cells or RAW264.7 cells were seeded on coverslips in 6-well plates, and handled with the same procedure described above. The concentration of schisandrin A used was $10 \mu \mathrm{M}$.

\subsection{Western blot assay}

Total protein lysates were extracted from each group of cells using ice-cold RIPA lysis buffer. The protein concentration of each sample was determined by BCA kit. The supernatant of each sample was boiled with $1 / 4$ volume of boiling $5 x$ loading buffer in a metal bath at $100{ }^{\circ} \mathrm{C}$ for $5 \mathrm{~min}$. For loading, a 20 mug total protein per sample was calculated and loaded onto sodium dodecyl sulfate polyacrylamide gel electrophoresis (SDS-PAGE), followed by wet transfer to a PVDF membrane. The membrane was blocked with $5 \%$ Bovine Serum Albumin (BSA, Sangon, China) $1 \mathrm{~h}$ at room temperature; primary antibodies were used: anti-iNOS (1:1000), anti-COX-2 (1:1000), anti-p-p65 (1:1000), anti-p65 (1:1000), anti-E-cadherin (1:1000), anti- $\beta$-catenin (1:1000), and anti-GAPDH (1:1000), overnight at $4^{\circ} \mathrm{C}$. All antibodies were purchased from Abcam (USA). Then, the corresponding secondary antibodies (1:4000) were added for 1 $\mathrm{h}$ at room temperature. Gray scale expression of protein bands was analyzed by Image $\mathrm{J}$ analysis software, and relative protein expression was obtained using GAPDH as the corresponding pair internal reference. 


\subsection{Immunofluorescence}

Cells cultured on glass slides were fixed with $4 \%$ paraformaldehyde for 20 min, permeabilized with $0.5 \%$ Triton X-100 for $15 \mathrm{~min}$, and then blocked with goat serum for $1 \mathrm{~h}$ at room temperature. Primary antibodies: anti-E-cadherin (1:200), anti- $\beta$-catenin (1:200), and anti-COX-2 (1:200) were added dropwise and incubated overnight at $4{ }^{\circ} \mathrm{C}$. Then, the cells were incubated with corresponding fluorescent secondary antibody (1:1000) for $1 \mathrm{~h}$ at room temperature in the dark. DAPI was used for nuclear staining for $10 \mathrm{~min}$. Immunofluorescence photography was performed using a super-resolution confocal microscope.

\subsection{Animals}

BALB/c male mice (8-12 weeks, 18-22 g) were obtained from the Laboratory Animal Center of Guangdong Medical University. Before the experiment, the mice were kept in a standard laboratory animal facility for 1 week. All mouse experiments were approved by the Institutional Animal Care and Use Committee of Guangdong Medical University.

\subsection{OVA-induced asthma mouse model and drug treatment}

The mice were randomly divided into three groups: control group, OVA group and OVA + schisandrin A (OVA + Sch A) group ( $n=7$ per group). On days 0,8 , and 15 , the OVA and OVA + Sch A groups were sensitized with an intraperitoneal injection (i.p.) of $0.2 \mathrm{ml}$ of sensitizing solution containing $50 \mu \mathrm{g}$ of ovalbumin (OVA, Sigma, USA), 100 ul of aluminum hydroxide (Thermo Scientific Pierce, USA) and $100 \mu$ l of saline. The control group was replaced with an equal volume of normal saline. Then, using an ultrasonic nebulizer (Omron Automation) on days 16-22, the mice in OVA and OVA + Sch A groups were airway challenged with $3 \%$ OVA solution (dissolved in PBS) for 30 min each time. The control group inhaled PBS instead. The OVA + Sch A group was intraperitoneal injection with schisandrin A (40 mg/ $\mathrm{kg}$, in $0.5 \%$ carboxymethylcellulose sodium aqueous solution) half an hour before nebulization, and the control and OVA groups were orally administered with an equal volume of carboxymethylcellulose sodium solution.

\subsection{Blood collection and flow cytometry}

Anesthetized mice were bled by retro-orbital puncture using heparinized capillary tube. Blood samples were placed in heparin sodium treated EP tubes, centrifuged at $3000 \mathrm{~g}$ for $5 \mathrm{~min}$ at $4{ }^{\circ} \mathrm{C}$, and the supernatant was discarded. The cells were re-suspended in RPMI 1640 medium containing $50 \mathrm{ng} / \mathrm{mL}$ phorbol 12-myristate 13-acetate (PMA) and $1 \mu \mathrm{g} / \mathrm{mL}$ ionomycin, mixed well and cultured for $5 \mathrm{~h}$ in the 37 ${ }^{\circ} \mathrm{C}$ and $5 \% \mathrm{CO} 2$ incubator. Cell pellets were treated with $1 \mathrm{X}$ red blood cell lysis buffer for 10 min on ice to remove any red blood cells and washed once with PBS. The cells were incubated with Fc-receptor blocking antibody (anti-CD16/32, Biolegend, USA) to reduce non-specific binding. Subsequently, the cells stained with the FITC anti-mouse CD3, PE anti-mouse CD4, Alexa fluor 700 anti-mouse CD8a and Brilliant Violet 650 anti-mouse CD25 antibodies for 20 minutes at $4{ }^{\circ} \mathrm{C}$ in the dark. Then, the cells were fixed and 
permeabilized, and further stained with PE anti-mouse IL-4 and PerCP/Cyanine5.5 anti-mouse IFN- $\gamma$ antibodies for 20 minutes at $4{ }^{\circ} \mathrm{C}$ in the dark. All antibodies were purchased from BioLegend (USA). The percentages of CD3 + CD $4+$ IFN- $y$ Th1 and CD3 + CD $4+$ IL-4 + Th2 cells were detected through flow cytometry.

\subsection{Collection and processing of bronchoalveolar lavage fluid (BALF)}

Twenty-four hours after the last OVA inhalation, mice were sacrificed after anesthesia by pentobarbital (50 mg/kg i.p.). BALF was collected three times from each mouse by endotracheal intubation with $0.8 \mathrm{ml}$ of ice-cold PBS. Cell numbers were counted using a hemocytometer. In order to distinguish differential cells, BALF was centrifuged at $800 \mathrm{~g}$ for 5 min using a centrifuge, and the supernatant was taken, resuspended with 100 ul PBS for precipitation, and dropped onto a glass slide. The cells were fixed with fixative and stained with Wright-Giemsa staining solution. 200 white blood cells were read under the microscope. The number of various white blood cells was counted and the percentage of various white blood cells were calculated.

\subsection{Lung tissue staining}

Lung tissue samples were made from the left lung which were harvested and fixed in $10 \%$ neutral formalin, dehydrated, embedded in paraffin. Lung tissues were cut into $5 \mu \mathrm{m}$ paraffin sections. The paraffin embedded tissue sections were baked at $60^{\circ} \mathrm{C}$ for 1 hour, deparaffinized and rehydrated in xylene and graded ethanol solutions. Hematoxylin and eosin (H \& E) staining was used to determine inflammatory cell infiltration and periodic acid Schiff (PAS) was used to evaluate mucus production.

\subsection{Immunohistochemistry}

After baking, deparaffinization, and hydration, paraffin sections were microwaved in citrate buffer $(\mathrm{pH}$ 6.0) for $10 \mathrm{~min}$, followed by cooling at room temperature. $5 \%$ goat serum was added dropwise to block the sections for 20 min at room temperature, and then a-SMA primary antibody (1:400, Abcam, USA) was incubated overnight at $4{ }^{\circ} \mathrm{C}$. Next, the corresponding secondary antibody was incubated for 1 hour at room temperature after 3 washes with PBS for $5 \mathrm{~min}$. The sections were developed with diaminobenzidine solution (DAB) for $7 \mathrm{~min}$, counterstained by hematoxylin for $2 \mathrm{~min}$, mounted, and visualized under a microscope.

\subsection{Data Presentation and Statistical Analysis}

All ata was presented as means \pm standard deviation (SD) of three independent experiments and analyzed by two-tailed Student t-test or one-way analysis of variance (ANOVA), followed by Tukey tests for multiple comparisons, when appropriate ( $p<0.05$ was considered as statistically significant).

\section{Results}




\subsection{Prediction of action targets of schisandrin A and screening of common targets}

The 2D and 3D structures of schisandrin A were obtained by searching PubChem database, as shown in Fig. 1A. In order to ensure the integrity of the target collection, we used Swisstarget Prediction and other databases to predict the potential targets, and combined with relevant literature reports, we obtained 99 targets of Schisandrin A (after deleting the repeat targets). 8162 targets of asthma were obtained from OMIM database and GeneCards database. The targets of Schisandrin A were mapped to the targets of asthma, and the Venn map (Fig. 1B) was drawn. After mapping, 68 intersection targets were obtained. The results show that the compound Schisandrin A may synergistically treat asthma through multiple potential action targets.

\subsection{Protein interaction network}

The PPI network (Fig. 1B) was obtained from STRING database analysis, including 68 nodes and 373 edges. After excluding the genes that are not associated with other genes, the obtained tsv file was imported into Cytoscape for network topology analysis, as shown in Fig. 1C. The analysis results show that the network contains 62 nodes and 373 edges, with clustering coefficient of 0.561 and network centralization of 0.440 . Each edge is short, and each gene has an average length of 12 . These data indicate that the target genes are all closely related. At the same time, through the above analysis, it is speculated that these cross genes may be potential therapeutic target genes of schisandrin $A$ in the treatment of asthma (Fig. 1C), reflecting the comprehensive regulation characteristics of schisandrin $A$ multi-target. Cytohubba is ranked according to the attributes of nodes in the network, and MCC is a new method, which has better performance in the prediction of key proteins in PPI network. As shown in Fig. 1D, through the MCC algorithm in the cytohubba plug-in, we obtained the top 20 key genes, namely TP53, AKT1, CASP3, TNF, IL6, PTGS2, STAT3, RELA, NFKBIA, IL1B, MMP9, TLR4, BCL2L1, SOD2, EGFR, NOS2, CCND1, IKBKB, MPO and NQ01. The detailed information is shown in Table 1. These genes may play a key role in the mechanism of schisandrin $A$ in the treatment of asthma, which can be considered as the core targets of schisandrin $A$ in the treatment of asthma. 
Table 1

Information of the top 20 key genes according to MCC algorithm.

\begin{tabular}{|c|c|c|c|c|c|c|}
\hline Rank & name & MCC score & Degree & Betweenness & Closeness & ClusteringCoefficient \\
\hline 1 & TP53 & 20200000000 & 34 & 223.0549 & 46.5 & 0.44207 \\
\hline 2 & AKT1 & 20200000000 & 38 & 735.1304 & 49.33333 & 0.34282 \\
\hline 3 & CASP3 & 20200000000 & 31 & 256.0898 & 45.5 & 0.47957 \\
\hline 4 & TNF & 20200000000 & 31 & 366.629 & 45.66667 & 0.47527 \\
\hline 5 & IL6 & 20200000000 & 32 & 221.2603 & 45.66667 & 0.46976 \\
\hline 6 & PTGS2 & 20200000000 & 30 & 252.2992 & 44.66667 & 0.47586 \\
\hline 7 & STAT3 & 20200000000 & 23 & 148.6305 & 41 & 0.67194 \\
\hline 8 & RELA & 19700000000 & 20 & 18.99462 & 39.33333 & 0.78421 \\
\hline 9 & NFKBIA & 19700000000 & 17 & 2.19322 & 37.33333 & 0.91912 \\
\hline 10 & IL1B & 19700000000 & 23 & 129.2368 & 41 & 0.64822 \\
\hline 11 & MMP9 & 19700000000 & 22 & 50.1608 & 40 & 0.68831 \\
\hline 12 & TLR4 & 19600000000 & 20 & 21.86364 & 39.66667 & 0.77895 \\
\hline 13 & BCL2L1 & 13500000000 & 22 & 38.08739 & 40.5 & 0.69697 \\
\hline 14 & SOD2 & 12900000000 & 20 & 20.54597 & 39 & 0.73158 \\
\hline 15 & EGFR & 6720000000 & 22 & 147.6082 & 40.16667 & 0.55844 \\
\hline 16 & NOS2 & 6710000000 & 17 & 18.78595 & 37.33333 & 0.80147 \\
\hline 17 & CCND1 & 527000000 & 18 & 19.88164 & 38.33333 & 0.75163 \\
\hline 18 & IKBKB & 519000000 & 14 & 6.70802 & 36 & 0.9011 \\
\hline 19 & MPO & 494000000 & 16 & 11.63371 & 37.33333 & 0.81667 \\
\hline 20 & NQ01 & 11200000 & 17 & 50.39787 & 37.33333 & 0.63235 \\
\hline
\end{tabular}

The 68 potential targets of schisandrin $A$ in the treatment of asthma were entered into DAVID database, and $\mathrm{P}<0.05$ was used as the screening condition. A total of $239 \mathrm{BP}$ (biological processes) enrichment results were mainly enriched in drug response, camp catabolism, negative regulation of apoptosis, positive regulation of nitric oxide biosynthesis, aging, lipoxygenase pathway, cell response to organic compounds, cGMP catabolism, inflammation and response to lipopolysaccharide. The chord diagram was drawn according to "term" and "gens", as shown in Fig. 2A. The enrichment results of CC (cell composition) and MF (molecular function) were 18 and 51, respectively. CC was mainly enriched in 
cytoplasm, membrane, organelle membrane, plasma membrane, voltage-gated calcium channel complex, and MF was mainly enriched in 3',5'-cyclic-nucleotide phosphodiesterase activity, 3',5'-cyclic-AMP phosphodiesterase activity, cAMP binding, 3',5'-cyclic-GMP phosphodiesterase activity, drug binding, identical protein binding, iron ion binding, oxidoreductase activity, enzyme binding, heme binding and so on. KEGG pathway is enriched in 81 signal pathways with statistical significance, as the top 20 pathways shown in the bubble diagram in Fig. 2C. KEGG is mainly enriched in hepatitis B, toxoplasmosis, apoptosis, legionellosis, tuberculosis, small cell lung cancer, pertussis, Chagas disease (American trypanosomias), TNF signaling pathway, NF-KB signaling pathway, CAMP signaling pathway, Pathways in cancer, Pancreatic cancer, HIF-1 signaling pathway, Leishmaniasis, Serotonergic synapse, MicroRNAs in cancer, Prostate cancer, Morphine addiction, Measles and others. The above analysis results suggests that Schisandrin A may be used to treat asthma through these processes and pathways.

\subsection{Effects of Schizandrin A on the HDM-induced inflammatory response in $16 \mathrm{HBE}$ cells}

To investigate the inflammatory response of schisandrin A on HDM-induced $16 \mathrm{HBE}$ epithelial cells, we evaluated the effect of schisandrin A at concentrations lower than $10 \mu \mathrm{M}$. As shown in Fig. 3, HDM stimulation induced up-regulation of iNOS and COX-2 expression in 16 HBE epithelial cells. However, pretreatment with schisandrin A attenuated the expression of both in a concentration-dependent manner. In addition, HDM phosphorylated and activated NF-KB p65 in $16 \mathrm{HBE}$ epithelial cells, and pretreatment with schisandrin $A$ attenuated the phosphorylation of NF-KBp65 in a concentration-dependent manner.

\subsection{SchizandrinA has a protective effect against HDM- induced epithelial mucosal barrier damage in $16 \mathrm{HBE}$ cells}

The integrity of the epithelial barrier, is the first line of defense against chronic airway inflammation. Western blotting analysis showed that HDM treatment did not affect the expression of E-cadherin or $\beta$ catenin in normal $16 \mathrm{HBE}$ cells (Fig. 4A-C). Immunofluorescence showed that HDM promoted delocalization of E-cadherin and $\beta$-catenin in 16 HBE cells and exhibited discontinuous diffusion from adjacent cell boundaries to the cytoplasm, this delocalization was reversed to varying degrees by the intervention of schisandrin A (Fig. 4D-4E).

\subsection{Effect of schizandrin A on inflammatory responses in LPS-stimulated RAW264.7 macrophages}

To investigate the inflammatory response of schisandrin A on LPS-induced RAW246.7 macrophages, we evaluated the effect of schisandrin A at concentrations lower than $10 \mu \mathrm{M}$. As shown in Fig. 5, LPS stimulation induced the expression of iNOS and COX-2 in RAW246.7 macrophages. However, pretreatment with schisandrin $A$ attenuated the expression of both in a concentration-dependent manner. In addition, immunofluorescence results showed that COX-2 fluorescence intensity in RAW264.7 
macrophages was upregulated by LPS, while pretreatment with schisandrin A effectively inhibited this effect.

\subsection{Effects of schisandrin A on OVA-exposed asthmatic mice.}

After 22 days of OVA sensitization and challenge, we assessed the severity of airway inflammation in the lungs (Fig. 6A). OVA inhalation significantly increased the number and percent of eosinophils in BALF as compared to the healthy control, and schisandrin $A$ treatment effectively reduced the increase in inflammatory cells (Fig. 6B). At the same time, schisandrin A could obviously inhibit the increase of Th2 cell ratio induced by OVA (Fig. 6C). In addition, OVA induction occurred corresponding pathological changes, including extensive inflammatory cell infiltration in the airways, excessive mucus secretion, and airway remodeling. The level of infiltration of inflammatory cells was assessed by HE staining of the lung, and the level of airway mucus secretion was measured by PAS staining. Compared with the control group, OVA treatment significantly increased inflammatory cell infiltration and mucus secretion, while schisandrin A effectively reduced inflammatory cell infiltration and the overproduction of mucus (Fig. 6D). Similarly, the expression of a-SMA, a major protein of airway remodeling in asthma, was analyzed using immunohistochemistry. We observed airway thickening and increased expression of aSMA in OVA-treated airways. However, schisandrin A significantly alleviated these pathological changes (Fig. 6D).

\section{Discussion}

Fructus Schisandrae Chinensis (FSC) is the dried and ripe fruit of Schisandra chinensis, which is a traditional Chinese herb originally recorded in Shen Nong Ben Cao Jing, and has antibacterial, liver protection, anti-tumor, antioxidant, sedative and hypnotic effects [19]. Schisandrin A belongs to the lignans class, the main characteristic active component of FSC, which has therapeutic effects on inflammatory diseases such as osteoarthritis, mastitis, and neuroninflammation [14, 20, 21]. The mechanism of action of schisandrin A has been found to be associated with inhibition of the NF-KB signaling pathway and inhibition of the expression levels of iNOS and COX-2 $[14,15,20]$. The pathogenesis of asthma is closely related to exposure to allergens, so chronic inflammation is aggravated once the airways are exposed to allergens [22]. Airway epithelial shedding and mucin production are increased in asthmatic patients, and exposure to allergens leads to increased NF-KB activation, further exacerbating epithelial barrier dysfunction and increasing the expression of proinflammatory factors $[23,24]$. In our study, we found that schisandrin A has a protective effect on dust mite-induced $16 \mathrm{HBE}$ epithelial cell barrier damage, in addition to an inhibitory effect on the phosphorylation of NF-KB p65.

Prostaglandin-endoperoxidase (PTGS) is a key enzyme in the prostaglandin biosynthetic pathway, of which, PTGS2 is the main isoenzyme responsible for the production of inflammatory prostaglandins and plays an important role in the inflammatory response $[25,26]$. The iNOS and COX-2 induce the generation 
of a large number of proinflammatory mediators such as nitric oxide (NO) and prostaglandin E2 (PGE2) during inflammation [27]. In the pathogenesis of asthma, the expression of COX-2 and PGE2 was upregulated, and at the same time, COX-2 has a regulatory effect on the differentiation of T lymphocytes [28-30]. In addition, it was found that NF-KB-induced iNOS and COX-2 are important mediators in the development of pulmonary inflammation [7, 31, 32]. In patients with asthma, increased production of macrophage-derived nitric oxide reflects the severity of asthma, and increased iNOS derived NO may contribute to the imbalance of oxidant and antioxidant pathways [33]. It has been shown that LPS induction can lead to activation of Th2 responses and asthma [34]. In our study, schisandrin A exerted an excellent effect by inhibiting the production of iNOS and COX-2 in 16 HBE cells and activated murine macrophages.

It is well-known that Th1/Th2 imbalance is one of the key factors contributing to the pathogenesis and severity of asthma $[35,36]$. The most common type of asthma, allergic asthma, is mediated by Th2 cells characterized by the accumulation of eosinophils, bronchial hyperreactivity, and airway remodeling [37]. Allergic asthma patients usually present with clinical manifestations such as cough, wheezing and dyspnea $[38,39]$. Our in vivo study confirmed that schisandrin A significantly reduced the OVA-induced increase of inflammatory cells, such as the proportion of Th2 cells, and the number and proportion of eosinophils, as well as significantly alleviated inflammatory cell infiltration in lung tissue. Hypersecretion of mucus in the airways is another important feature of asthma. In severe asthma, goblet cells are significantly increased, and metaplasia and hyperplasia occur, which leads to mucin overproduction in the airways [40,41]. In this study, schisandrin A significantly alleviated OVA-induced mucus hypersecretion and goblet cell hyperplasia. In addition, studies have shown that a-SMA is associated with airway remodeling and severity in asthma $[42,43]$. Schisandrin $A$ was also effective in reducing a-SMA production. According to these results, we believe that schisandrin A has a therapeutic effect on asthma.

\section{Conclusion}

In this study, we proved that schisandrin A significantly inhibited the inflammatory response of $16 \mathrm{HBE}$ epithelial cells induced by HDM and RAW264.7 cells induced by LPS, respectively, and significantly reduced OVA-induced airway inflammation and mucus hypersecretion of airway goblet cells in mice. In conclusion, the results suggested that Schisandrin A can reduce asthma symptoms by inhibiting inflammation production, including lowering the Th2 cell ratio, which provides a basis for further understanding of the treatment of asthma with Schisandrin $A$.

\section{Abbreviations}

TCM Traditional Chinese medicines

NF-kB signaling pathway Factor-kappaB signaling pathway COX-2 Cyclooxygenase 2 
Th2 T helper type 2

PTGS2 Prostaglandin-endoperoxide synthase 2

PPI Protein-protein interaction

GO Gene Ontology

KEGG Kyoto Encyclopedia of Genes and Genomes

HDM D. pteronyssinus

LPS Lipopolysaccharide

OVA Ovalbumin

FSC Fructus Schisandrae Chinensis

\section{Declarations}

\section{Author Contributions}

LX Luo, YG Huang conceived and designed the review. Q Qiu, LX Luo, JT Su wrote the manuscript. Q Qiu, FF Huang and JT Su carried out the experiment. LX Luo and YG Huang reviewed the paper and provided comments. All authors contributed to the article and approved the submitted version.

\section{Acknowledgment}

The authors acknowledge the the Affiliated Hospital of Guangdong Medical University Medical Center Flow Cytometry core for acquiring the flow cytometry data presented in this manuscript.

\section{Funding}

This project was supported by Administration of Traditional Chinese Medicine of Guangdong Province (20201180); Administration of Traditional Chinese Medicine of Guangdong Province (20211223); Science and Technology Special Project of Zhanjiang (2019A01009); Basic and Applied Basic Research Program of Guangdong Province (2019A1515110201); Program of Department of Natural Resources of Guangdong Province (No. GDNRC [2020]038 and [2021]53); Discipline Construction Project of Guangdong Medical University (4SG21004G).

\section{Declaration of Competing Interest}

The authors declare that they have no known competing financial interests or personal relationships that could have appeared to influence the work reported in this paper.

\section{Availability of data and materials}

Page 12/21 
The data used to support the findings of this study are included within the article.

\section{Declaration of competing interest}

The authors declare that they have no competing interests.

\section{Consent for publication}

Not applicable.

\section{Ethics approval and consent to participate}

All animal experimental procedures were approved by the Animal Care and Use Committee of Guangdong Medical University in line with the guidelines of National and Institutional Guidelines for Animal Care

\section{References}

1. Georas SN, Rezaee F. Epithelial barrier function: at the front line of asthma immunology and allergic airway inflammation. J Allergy Clin Immunol. 2014;134:509-20. doi:10.1016/j.jaci.2014.05.049.

2. Zhu T, Zhang W, Wang DX, Huang NW, Bo H, Deng W, et al. Rosuvastatin attenuates mucus secretion in a murine model of chronic asthma by inhibiting the gamma-aminobutyric acid type $A$ receptor. Journal. 2012;125:1457-64.

3. Gurusamy M, Nasseri S, Lee H, Jung B, Lee D, Khang G, et al. Kinin B1 receptor antagonist BI113823 reduces allergen-induced airway inflammation and mucus secretion in mice. Pharmacol Res. 2016;104:132-9. doi:10.1016/j.phrs.2015.12.017.

4. Mishra V, Banga J, Silveyra P. Oxidative stress and cellular pathways of asthma and inflammation: Therapeutic strategies and pharmacological targets. Pharmacol Ther. 2018;181:169-82. doi:10.1016/j.pharmthera.2017.08.011.

5. Ghosh S, Hayden MS. New regulators of NF-kappaB in inflammation. Nat Rev Immunol. 2008;8:83748. doi:10.1038/nri2423.

6. Edwards MR, Bartlett NW, Clarke D, Birrell M, Belvisi M, Johnston SL. Targeting the NF-kappaB pathway in asthma and chronic obstructive pulmonary disease. Pharmacol Ther. 2009;121:1-13. doi:10.1016/j.pharmthera.2008.09.003.

7. Athari SS. Targeting cell signaling in allergic asthma. Signal Transduct Target Ther. 2019;4:45. doi:10.1038/s41392-019-0079-0.

8. Sheller JR, Polosukhin VV, Mitchell D, Cheng DS, Peebles RS, Blackwell TS. Nuclear factor kappa B induction in airway epithelium increases lung inflammation in allergen-challenged mice. Exp Lung Res. 2009;35:883-95. doi:10.3109/01902140903019710. 
9. Schuliga M. NF-kappaB Signaling in Chronic Inflammatory Airway Disease. Biomolecules. 2015;5:1266-83. doi:10.3390/biom5031266.

10. Richards LB, Li M, Folkerts G, Henricks PAJ, Garssen J, van Esch BCAM. Butyrate and Propionate Restore the Cytokine and House Dust Mite Compromised Barrier Function of Human Bronchial Airway Epithelial Cells. Int J Mol Sci. 2020;22:65. doi:10.3390/ijms22010065.

11. Boulet LP, Reddel HK, Bateman E, Pedersen S, FitzGerald JM, O'Byrne PM. The Global Initiative for Asthma (GINA): 25†நyears later. Eur Respir J. 2019;54:2. doi:10.1183/13993003.00598-2019.

12. Cui L, Zhu W, Yang Z, Song X, Xu C, Cui Z, et al. Evidence of anti-inflammatory activity of Schizandrin $A$ in animal models of acute inflammation. Naunyn Schmiedebergs Arch Pharmacol. 2020;393:2221-9. doi:10.1007/s00210-020-01837-x.

13. Qiu F, Liu H, Duan H, Chen P, Lu SJ, Yang GZ, et al. Isolation, Structural Elucidation of Three New Triterpenoids from the Stems and Leaves of Schisandra chinensis (Turcz) Baill. Molecules. 2018;23:1624. doi:10.3390/molecules23071624.

14. Tu C, Huang $X$, Xiao Y, Song M, Ma Y, Yan J, et al. Schisandrin A Inhibits the IL-1 $\beta$-Induced Inflammation and Cartilage Degradation via Suppression of MAPK and NF-KB Signal Pathways in Rat Chondrocytes. Front Pharmacol. 2019;10:41. doi:10.3389/fphar.2019.00041.

15. Kwon DH, Cha HJ, Choi EO, Leem SH, Kim GY, Moon SK, et al. Schisandrin A suppresses lipopolysaccharide-induced inflammation and oxidative stress in RAW 264.7 macrophages by suppressing the NF-KB, MAPKs and PI3K/Akt pathways and activating Nrf2/HO-1 signaling. Int J Mol Med. 2018;41:264-74. doi:10.3892/ijmm.2017.3209.

16. Lee MY, Seo CS, Lee NH, Ha H, Lee JA, Lee H, et al. Anti-asthmatic effect of schizandrin on OVAinduced airway inflammation in a murine asthma model. Int Immunopharmacol. 2010;10:1374-9. doi:10.1016/j.intimp.2010.07.014.

17. Moon PD, Jeong HJ, Kim HM. Effects of schizandrin on the expression of thymic stromal lymphopoietin in human mast cell line HMC-1. Life Sci. 2012;91:384-8. doi:10.1016/j.lfs.2012.08.009.

18. Zhang XY, Tang XY, Ma LJ, Guo YL, Li XS, Zhao LM, et al. Schisandrin B down-regulated IncRNA BCYRN1 expression of airway smooth muscle cells by improving miR-150 expression to inhibit the proliferation and migration of ASMC in asthmatic rats. Cell Prolif. 2017;50:e12382. doi:10.1111/cpr.12382.

19. Panossian A, Wikman G. Pharmacology of Schisandra chinensis Bail.: an overview of Russian research and uses in medicine. J Ethnopharmacol. 2008;118:183-212. doi:10.1016/j.jep.2008.04.020.

20. Song F, Zeng K, Liao L, Yu Q, Tu P, Wang X. Schizandrin A Inhibits Microglia-Mediated Neuroninflammation through Inhibiting TRAF6-NF-KB and Jak2-Stat3 Signaling Pathways. PLoS ONE. 2016;11:e0149991. doi:10.1371/journal.pone.0149991.

21. Xu D, Liu J, Ma H, Guo W, Wang J, Kan X, et al. Schisandrin A protects against lipopolysaccharideinduced mastitis through activating Nrf2 signaling pathway and inducing autophagy. Int 
Immunopharmacol. 2020;78:105983. doi:10.1016/j.intimp.2019.105983.

22. Lambrecht BN, Hammad H. Allergens and the airway epithelium response: gateway to allergic sensitization. J Allergy Clin Immunol. 2014;134:499-507. doi:10.1016/j.jaci.2014.06.036.

23. Holgate ST, Davies DE, Puddicombe S, Richter A, Lackie P, Lordan J, et al. Mechanisms of airway epithelial damage: epithelial-mesenchymal interactions in the pathogenesis of asthma. Eur Respir $\mathrm{J}$ Suppl. 2003;44:24s-29s. doi:10.1183/09031936.03.00000803.

24. Rahman I, Gilmour PS, Jimenez LA, MacNee W. Oxidative stress and TNF-alpha induce histone acetylation and NF-kappaB/AP-1 activation in alveolar epithelial cells: potential mechanism in gene transcription in lung inflammation. Journal. 2002;234-235:239-48.

25. Bukata SV, Gelinas J, Wei X, Rosier RN, Puzas JE, Zhang X, et al. PGE2 and IL-6 production by fibroblasts in response to titanium wear debris particles is mediated through a Cox-2 dependent pathway. J Orthop Res. 2004;22:6-12. doi:10.1016/s0736-0266(03)00153-0.

26. Echizen K, Hirose O, Maeda Y, Oshima M. Inflammation in gastric cancer: Interplay of the COX2/prostaglandin E2 and Toll-like receptor/MyD88 pathways. Cancer Sci. 2016;107:391-7. doi:10.1111/cas.12901.

27. Posadas I, Terencio MC, Guillén I, Ferrándiz ML, Coloma J, Payá M, et al. Co-regulation between cyclo-oxygenase-2 and inducible nitric oxide synthase expression in the time-course of murine inflammation. Naunyn Schmiedebergs Arch Pharmacol. 2000;361:98-106. doi:10.1007/s002109900150.

28. Herrerias A, Torres R, Serra M, Marco A, Pujols L, Picado C, et al. Activity of the cyclooxygenase 2prostaglandin-E prostanoid receptor pathway in mice exposed to house dust mite aeroallergens, and impact of exogenous prostaglandin E2. J Inflamm (Lond). 2009;6:30. doi:10.1186/1476-9255-6-30.

29. Li H, Edin ML, Bradbury JA, Graves JP, DeGraff LM, Gruzdev A, et al. Cyclooxygenase-2 inhibits T helper cell type 9 differentiation during allergic lung inflammation via down-regulation of IL-17RB. Am J Respir Crit Care Med. 2013;187:812-22. doi:10.1164/rccm.201211-20730C.

30. Carey MA, Germolec DR, Bradbury JA, Gooch RA, Moorman MP, Flake GP, et al. Accentuated T helper type 2 airway response after allergen challenge in cyclooxygenase-1-/- but not cyclooxygenase-2-/mice. Am J Respir Crit Care Med. 2003;167:1509-15. doi:10.1164/rccm.200211-13830C.

31. Chen GG, Lee TW, Xu H, Yip JH, Li M, Mok TS, et al. Increased inducible nitric oxide synthase in lung carcinoma of smokers. Cancer. 2008;112:372-81. doi:10.1002/cncr.23166.

32. Anto RJ, Mukhopadhyay A, Shishodia S, Gairola CG, Aggarwal BB. Cigarette smoke condensate activates nuclear transcription factor-kappaB through phosphorylation and degradation of IkappaB(alpha): correlation with induction of cyclooxygenase-2. Carcinogenesis. 2002;23:1511-8. doi:10.1093/carcin/23.9.1511.

33. Prado CM, Martins MA, Tibério IF. Nitric oxide in asthma physiopathology. ISRN Allergy. 2011;2011:832560. doi:10.5402/2011/832560.

34. Piggott DA, Eisenbarth SC, Xu L, Constant SL, Huleatt JW, Herrick CA, et al. MyD88-dependent induction of allergic Th2 responses to intranasal antigen. J Clin Invest. 2005;115:459-67. 
doi:10.1172/jci22462.

35. Kay AB. Allergy and allergic diseases. First of two parts. N Engl J Med. 2001;344:30-7. doi:10.1056/nejm200101043440106.

36. Thorburn AN, Hansbro PM. Harnessing regulatory T cells to suppress asthma: from potential to therapy. Am J Respir Cell Mol Biol. 2010;43:511-9. doi:10.1165/rcmb.2009-0342TR.

37. Lambrecht BN, Hammad H, Fahy JV. The Cytokines of Asthma. Immunity. 2019;50:975-91. doi:10.1016/j.immuni.2019.03.018.

38. Kulkarni NS, Hollins F, Sutcliffe A, Saunders R, Shah S, Siddiqui S, et al. Eosinophil protein in airway macrophages: a novel biomarker of eosinophilic inflammation in patients with asthma. J Allergy Clin Immunol. 2010;126:61-9.e63. doi:10.1016/j.jaci.2010.03.026.

39. Holgate ST, Wenzel S, Postma DS, Weiss ST, Renz H, Sly PD. Asthma. Nat Rev Dis Primers. 2015;1:15025. doi:10.1038/nrdp.2015.25.

40. Ma J, Rubin BK, Voynow JA. Mucins, Mucus, and Goblet Cells. Chest. 2018;154:169-76. doi:10.1016/j.chest.2017.11.008.

41. Kudo M, Ishigatsubo Y, Aoki I. Pathology of asthma. Front Microbiol. 2013;4:263. doi:10.3389/fmicb.2013.00263.

42. Shipe R, Burdick MD, Strieter BA, Liu L, Shim YM, Sung SS, et al. Number, activation, and differentiation of circulating fibrocytes correlate with asthma severity. J Allergy Clin Immunol. 2016;137:750-7.e753. doi:10.1016/j.jaci.2015.07.037.

43. Dong L, Wang Y, Zheng T, Pu Y, Ma Y, Qi X, et al. Hypoxic hUCMSC-derived extracellular vesicles attenuate allergic airway inflammation and airway remodeling in chronic asthma mice. Stem Cell Res Ther. 2021;12:4. doi:10.1186/s13287-020-02072-0.

\section{Figures}


A
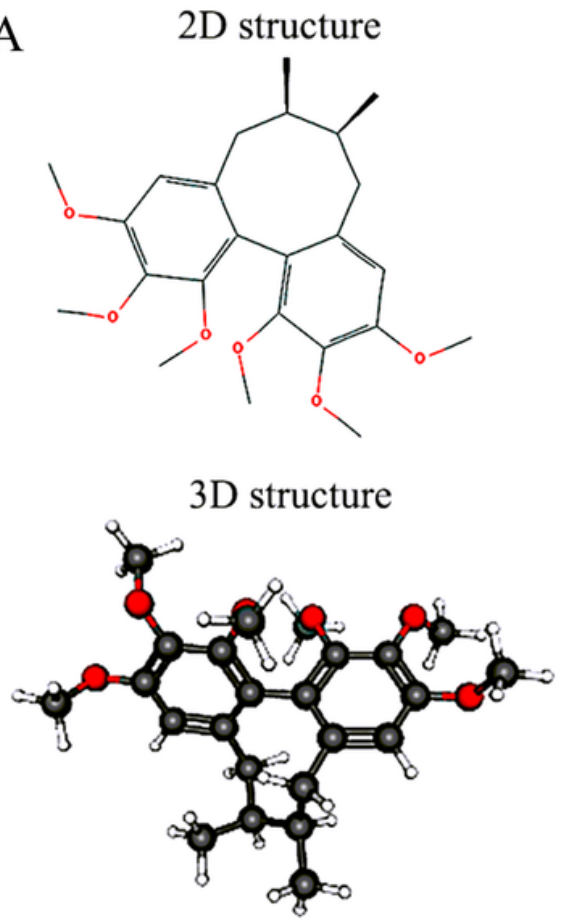

$\mathrm{B}$

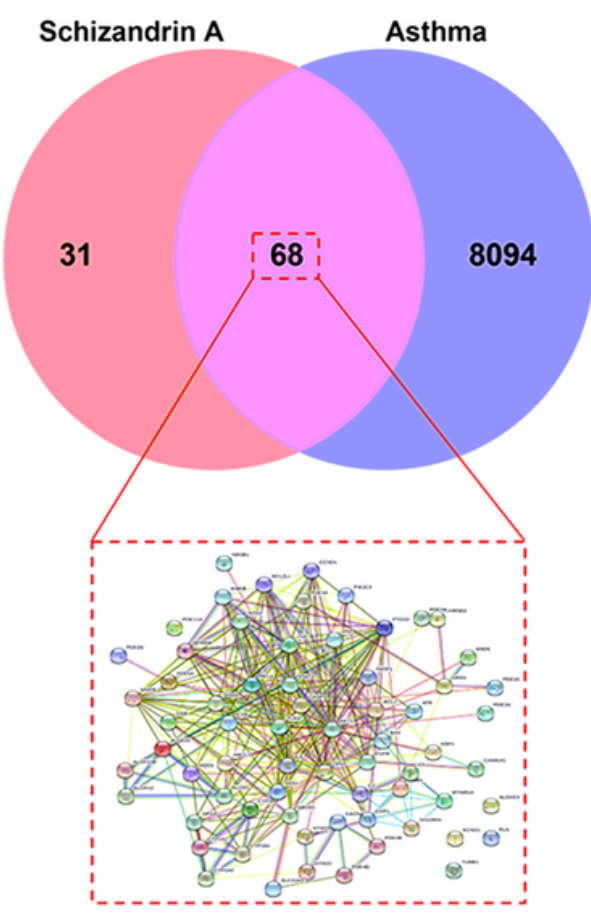

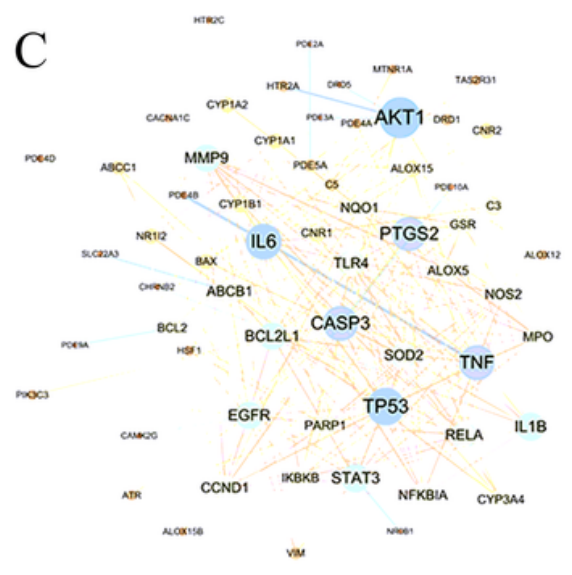

$\mathrm{D}$

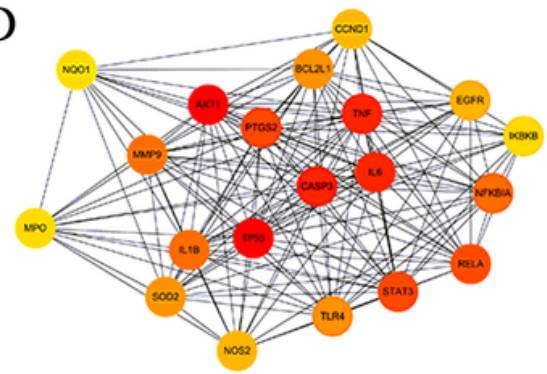

Figure 1

Relevant information on Schisandrin A and PPI network of its potential targets for asthma treatment. (A) 2D and 3D structures of Schisandrin A. (B) Venn diagram of targets acting on Schisandrin A and asthma. (C) Topological analysis of PPI network of potential targets of Schisandrin A in the treatment of asthma. (D) The network diagram of the top 20 key genes obtained from PPI network according to the MCC algorithm.
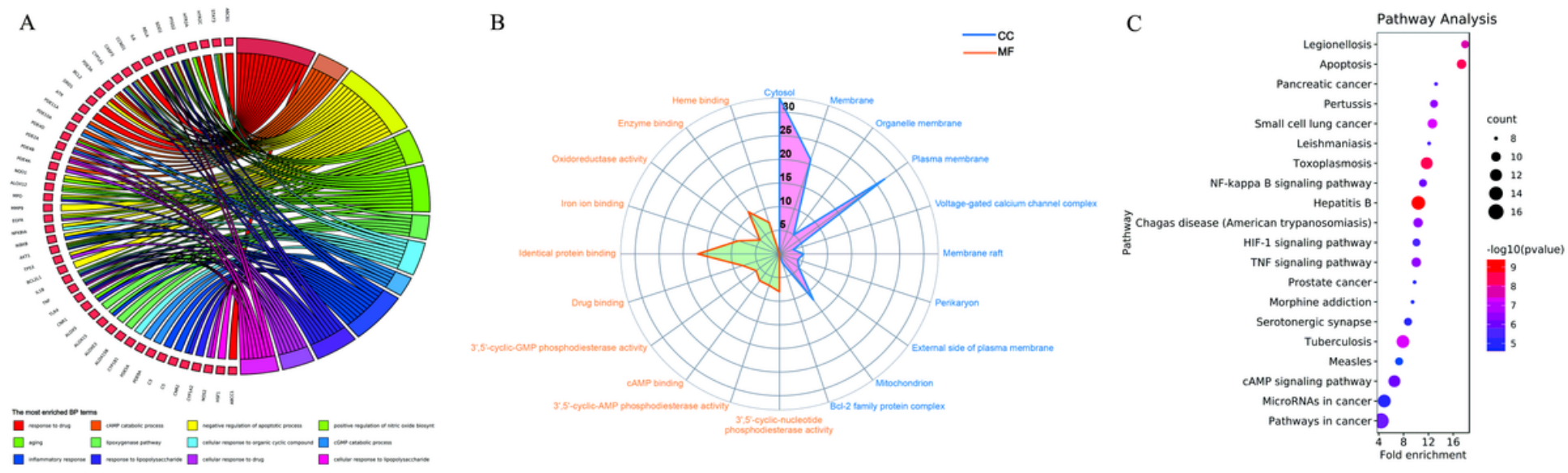

Figure 2

Enrichment analysis results of 68 potential targets of Schisandrin A in the treatment of asthma. (A) Chord diagram of the first $12 \mathrm{BP}$ enrichment analysis results. (B) The radar image of the first 10 enrichment results of CC and MF. (C) The first 20 KEGG signaling pathways shown in bubble chart. 
A

$\operatorname{HDM}(50 \mu \mathrm{g} / \mathrm{ml}) \quad 0++++$ Schisandrin A $(\mu \mathrm{M}) \quad 0 \quad 0 \quad 0 \quad 1 \quad 5 \quad 10 \quad \mathrm{kDa}$

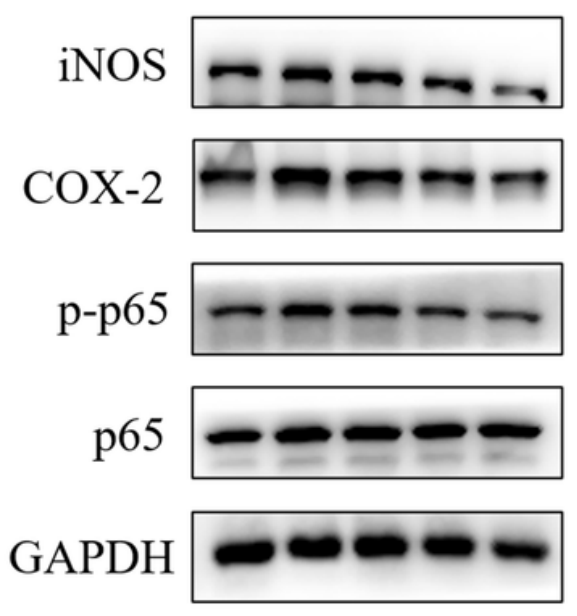

131

72

65

65

37
B

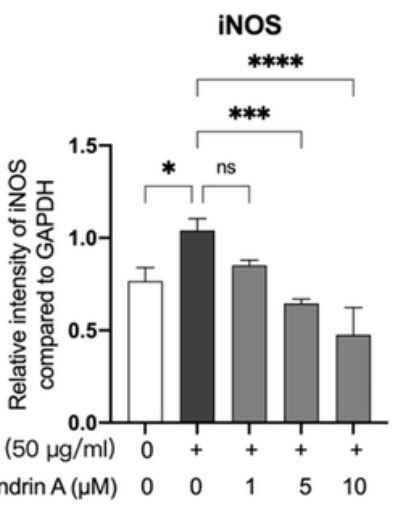

D

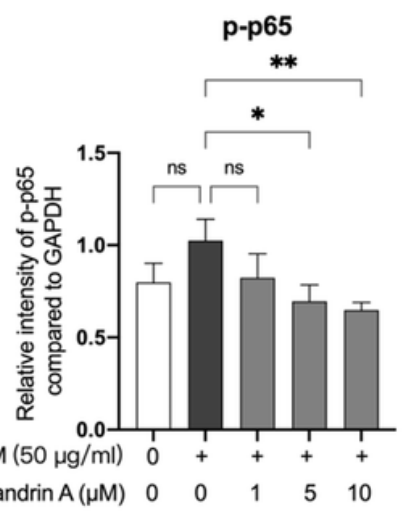

C

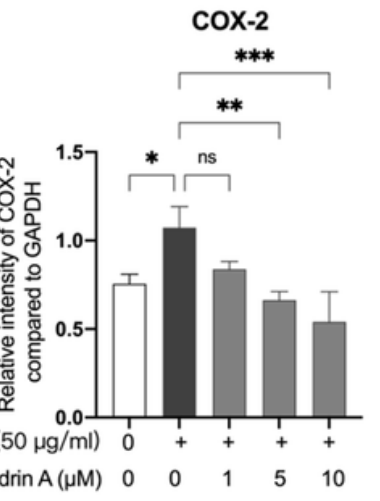

E
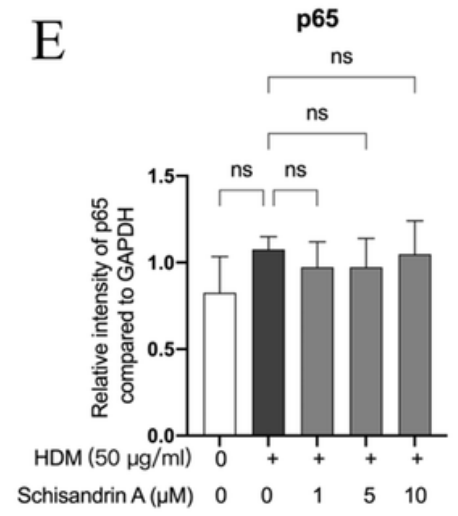

Figure 3

Effects of Schizandrin A on the HDM-induced inflammatory response in 16HBE epithelial cells. (Fig. A-E) $16 \mathrm{HBE}$ cells were pretreated with schisandrin $\mathrm{A}(1,5,10 \mu \mathrm{M})$ for $0.5 \mathrm{~h}$ and then treated with $50 \mu \mathrm{g} / \mathrm{mL}$ HDM for $24 \mathrm{~h}$. Protein expression of iNOS, (COX) -2, p-p65, and p65 were determined by western blotting, and quantified using Image $\mathrm{J}$ software. All the experiments were independently repeated at least three times. $\left({ }^{\star} p<0.05,{ }^{* \star} p<0.005,{ }^{* \star \star} p<0.0005,{ }^{* \star * *} p<0.0001\right.$, one-way ANOVA t-test) . 
A

$$
\operatorname{HDM}(50 \mu \mathrm{g} / \mathrm{ml}) \quad 0++++
$$

Schisandrin A $(\mu \mathrm{M}) \quad 0 \quad 0 \quad 0 \quad 1 \quad \begin{array}{lllll}10 & \mathrm{kDa}\end{array}$

E-cadherin

$\beta$-catenin

GAPDH

D

DAPI

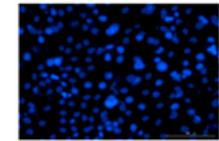

Control
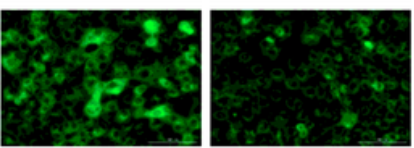

E-cadherin

Merge

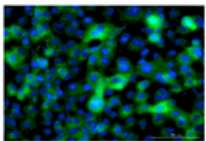

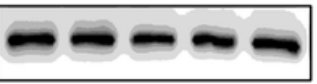
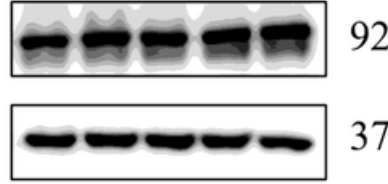

$\mathrm{HDM}+$

HDM Schisandrin A

B 125

92

37

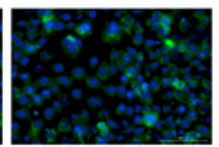

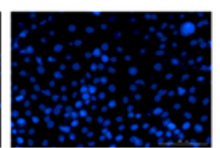

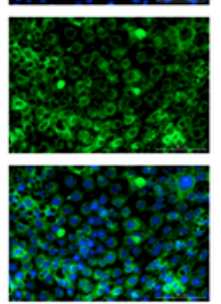

E

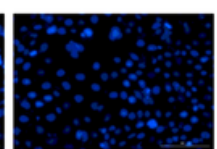

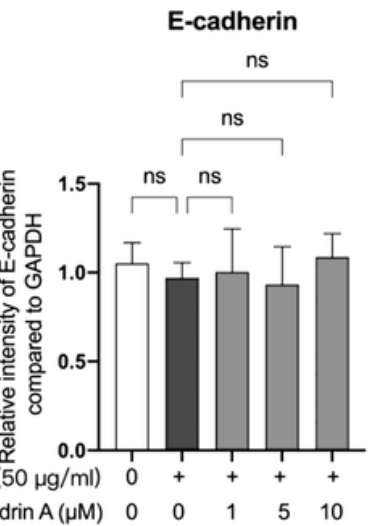

Control

DAPI
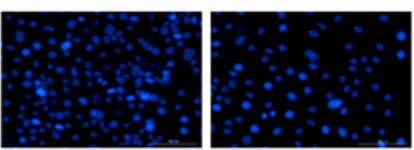

$\beta$-catenin
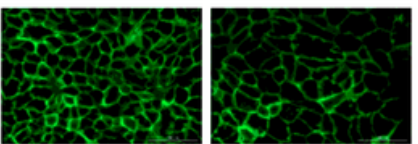

Merge

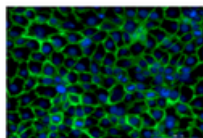

C

$\beta$-catenin

ns

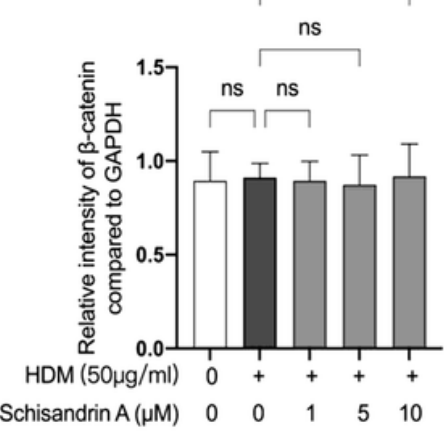

$\mathrm{HDM}+$

Schisandrin A

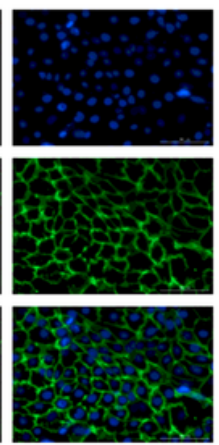

\section{Figure 4}

Schizandrin A has a protective effect against HDM-induced epithelial mucosal barrier damage in $16 \mathrm{HBE}$ cells. (A-C) $16 \mathrm{HBE}$ cells were pretreated with schisandrin $\mathrm{A}(1,5,10 \mu \mathrm{M})$ for $0.5 \mathrm{~h}$ and then treated with $50 \mu \mathrm{g} / \mathrm{mL}$ HDM for $24 \mathrm{~h}$. Western blotting measurements showed no difference in the total expression of E-cadherin and $\beta$-catenin protein, which was quantified using Image $J$ software. $16 \mathrm{HBE}$ cells were pretreated with schisandrin $A(10 \mu \mathrm{M})$ for $0.5 \mathrm{~h}$ and then treated with $50 \mu \mathrm{g} / \mathrm{mL}$ HDM for $24 \mathrm{~h}$. (D, E) Immunofluorescence showed that the distribution of E-cadherin and $\beta$-catenin protein was diffusely distributed from the membrane to the interstitial space (original magnification $\times 200$; scale bar $=100 \mu \mathrm{m}$; representative images from three experiments). All the experiments were independently repeated at least three times. (ns p $>0.05$, one-way ANOVA t-test) . 


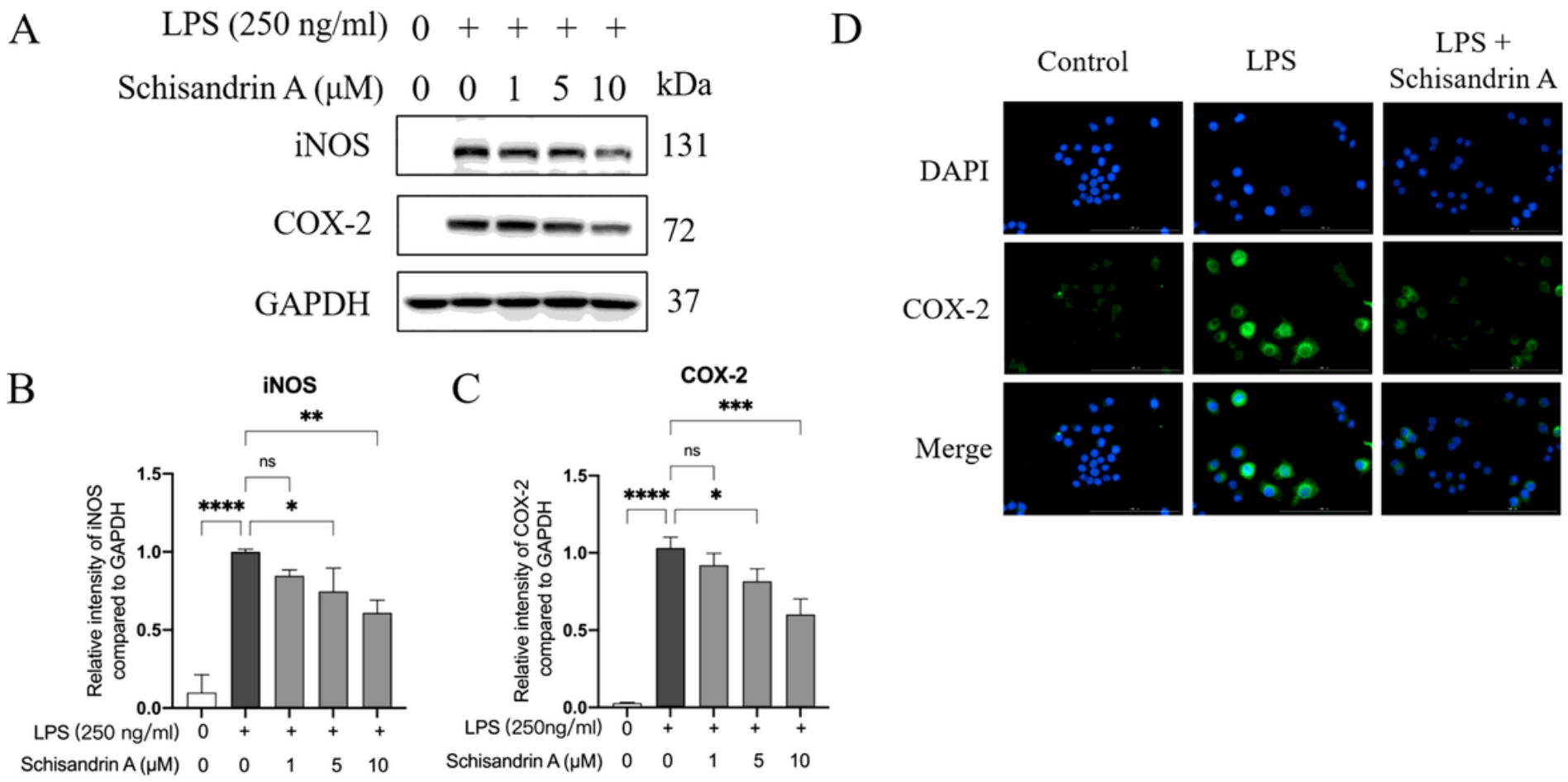

\section{Figure 5}

Schizandrin A inhibited the expression of iNOS and COX-2 proteins in LPS-stimulated RAW264.7 cells. (AC) RAW264.7 macrophages were pretreated with schisandrin $A(1,5,10 \mu \mathrm{M})$ for $0.5 \mathrm{~h}$ and then treated with $250 \mathrm{ng} / \mathrm{mL}$ LPS for $24 \mathrm{~h}$. The expression levels of iNOS and COX-2 proteins were measured by Western blotting and quantified using Image $\mathrm{J}$ software. All the experiments were independently repeated at least three times. $\left({ }^{*} p<0.05,{ }^{*} p<0.005,{ }^{* \star} p<0.0005,{ }^{* \star *} p<0.0001\right.$, one-way ANOVA t-test $\mathbb{R}$. (D) Distribution and expression of COX-2 by immunofluorescence. (original magnification $\times 200$; scale bar = 100 microm; representative images from three experiments). 


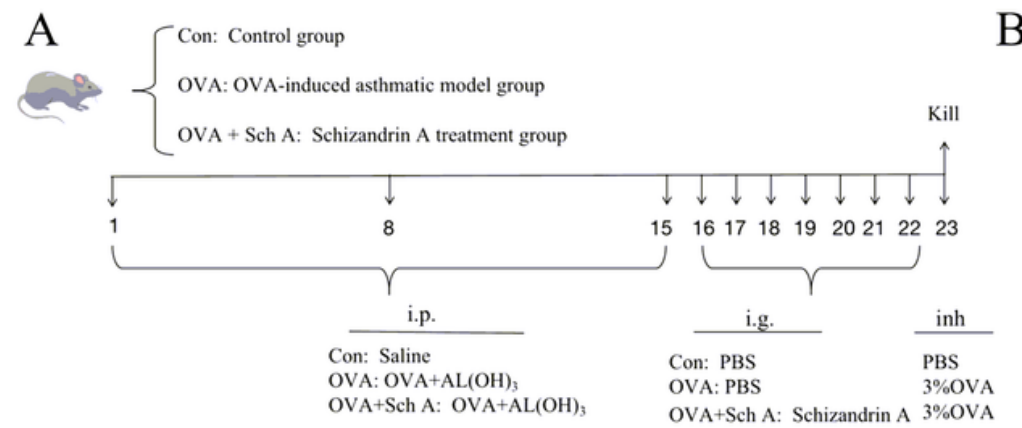

B

C

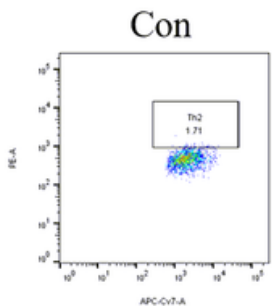

OVA+Sch A

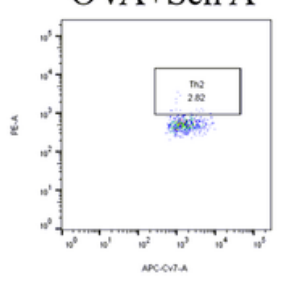

OVA
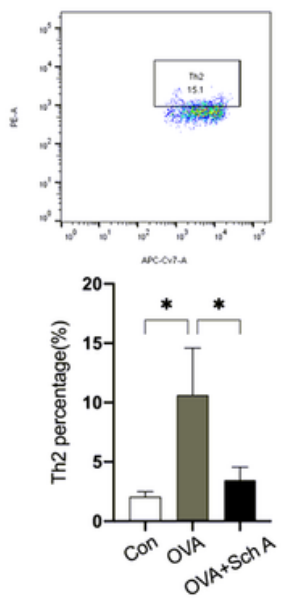

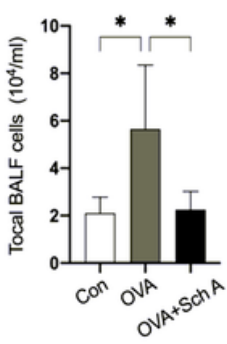

D Control
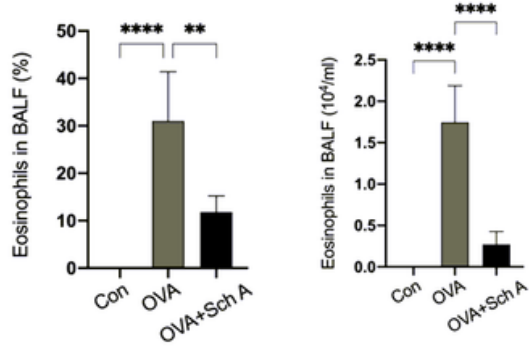

OVA

$\mathrm{OVA}+\mathrm{Sch} \mathrm{A}$
$\mathrm{H} \& \mathrm{E}$

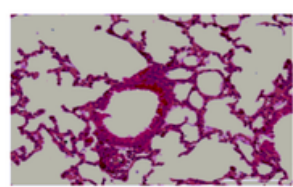

PAS
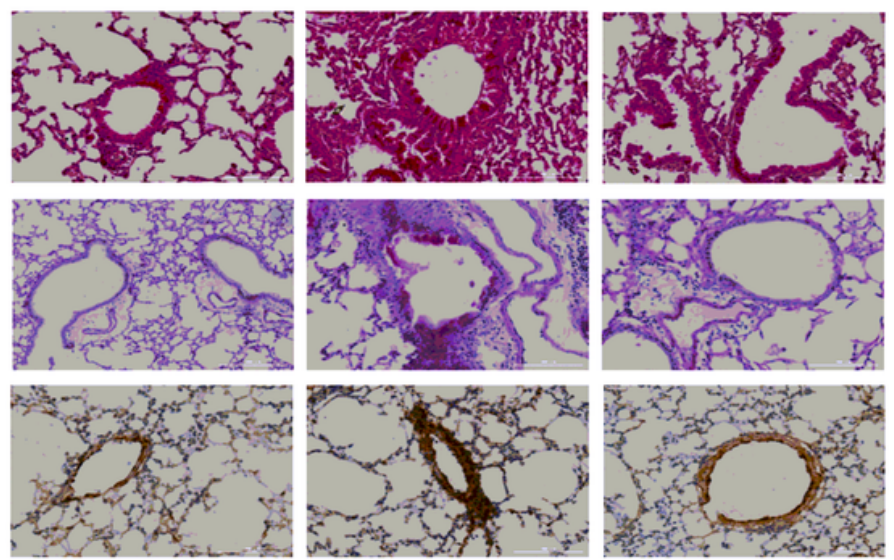

$\alpha$-SMA

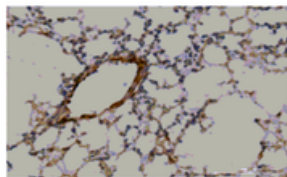

Figure 6

SchizandrinA reduced OVA-induced inflammation in mice and mucus secretion in the lungs of mice. (A) Experimental grouping and experimental process of OVA-induced asthma mice model. (B)To measure the number of total inflammatory cells and eosinophils in the bronchoalveolar lavage fluid. (C) The percentage of Th2 cells in the blood of mice was measured by flow cytometry. (D) $5 \mu \mathrm{m}$-thick lung sections from each group were taken for hematoxylin-eosin staining (H\&E) and periodic acid-Schiff (PAS). In addition, the expression of a-SMA was detected using immunohistochemistry. Staining to observe inflammatory infiltration and mucus secretion (original magnification x200; scale bar $=100 \mu \mathrm{m}$ ). 